\title{
A SINGLE COLUMN CHIROPTICAL HPLC BATCH ANALYSIS OF TEN AROMATIC ALCOHOLS
}

\author{
MARCELO A. MUNOOZ ${ }^{*}$; MARÍA-PAZ ORELLANA ${ }^{2}$, DANIELA POBLETE ${ }^{2}$
}

${ }^{1}$ Instituto de Ciencias Quimicas, Facultad de Ciencias, Universidad Austral de Chile, Casilla 567, Valdivia, Chile.
${ }^{2}$ Escuela de Quimica y Farmacia, Facultad de Ciencias, Universidad Austral de Chile, Casilla 567, Valdivia, Chile.

\section{ABSTRACT}

The HPLC chiral resolution of ten chiral aromatic alcohols were screened using six different polysaccharides-based chiral stationary phases (CSP) and under four normal phase conditions, using UV absorbance as detection. The screening showed that all compounds can be baseline resolved in at least one of the tested conditions, being the cellulose-methylbenzoate CSP the one with the best performance by resolving the full set. Furthermore, the use of two chiroptical detectors, based in optical rotation and electronic circular dichroism, permitted the ascertain of the elution order for each of the baseline separations. Finally, several options for a single-column batch analysis, in which samples of all compounds are tested for enantiomeric excess and absolute configuration, are proposed to analyze samples from the asymmetric reduction of the corresponding ketones. These options vary in the number of injections, analysis time and reliability, and their choice will mostly depend on the hardware and standards availability.

Keywords: HPLC-OR, HPLC-CD, aromatic alcohols, optical rotation detection, circular dichroism detection.

\section{INTRODUCTION}

The resolution and analysis of chiral compounds have become a major subject of attention in the research and development of new substances for a wide range of commercial applications ${ }^{1}$. Particularly, the pharmaceutical industry is now fully aware of the critical importance of the stereochemistry of chiral drugs since the safety issues that appeared during the second half of the past century ${ }^{2}$, but also more recently from the commercial advantages of producing enantiomerically pure compounds, also known as the "chiral switch" then, the need of enantioselective synthesis reactions in the manufacturing of chiral drugs has seen a marked increase, and the development of such reactions is now a major field of research. One of the most commonly used of these reactions is the enantioselective reduction of asymmetric ketones leading to chiral alcohols. Such reactions are often achieved through the use of chiral complexes of transition metals as catalysts during homogeneous hydrogenation ${ }^{4,5}$. Nevertheless, during the testing of these new catalysts it is crucial to be able to make rapid and precise measurements of the stereoselectivity achieved over a set of standard ketones. Most commonly, such procedure will include purification and subsequent assessment of enantiomeric excess (e.e.) using chiral GC or HPLC. However, a second step is needed to ascertain the absolute configuration (AC) of the preferred isomer since this cannot be inferred from the chromatographic run without the corresponding enantiomeric standards, and therefore is typically performed through a separate optical rotation measurement and comparison with literature data ${ }^{6,7}$. This two-step method can prove to be very time-consuming, particularly when a large combination of reaction parameters is being tested. Alternatively, chiral HPLC analysis coupled with chiroptical detection ${ }^{8}$ has the potential to ascertain the e.e. and AC of the reaction products in a single procedure, which in turn can be easily batched to obtain a highly automated multi-sample method. To such chiral separations, the combination of normal phase with amylose and cellulose based chiral stationary phases (CSP) are the more commonly used chromatographic conditions, and have been applied over a diverse range of substances ${ }^{9}$, including several chiral alcohols obtained in these studies ${ }^{10}$. However, only scattered information of the chromatographic conditions needed for these analyses can be found in the literature, and no efforts has been made to found the best combination of these conditions for a complete set of these compounds.
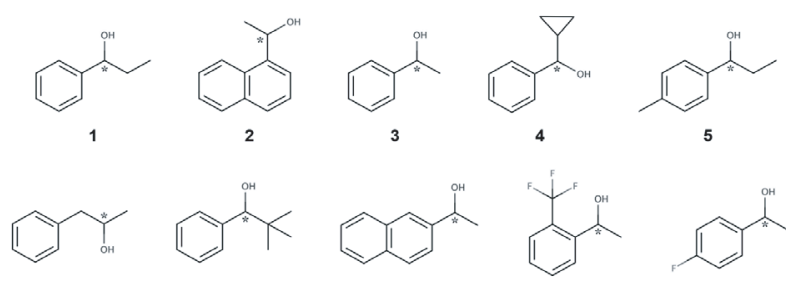

8

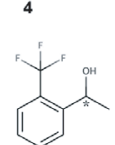

9

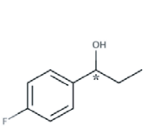

10 marked.

Fig. 1. Structures of aromatic alcohols 1-10. Asymmetric carbons have been

In the following article, the separation parameters obtained for ten aromatic alcohols shown in Figure 1 (1-10), commonly used in the development of stereoselective catalytic hydrogenation reactions, are presented. The chiral screening included six commercially available CSP operated under normal phase conditions using hexane with four different concentrations of iso-propanol as mobile phases. Additionally, the use of a laser-polarimetric detector arranged in tandem with the UV detector, permitted the ascertain of the elution order for each successful separation. Also, the use of a circular dichroism (CD) detector as an alternative to the UV-OR array is evaluated. Finally, based on the findings of the chiral screening and the chromatographic hardware available, several options for a single-column batch analysis in which the full compound set can be tested for e.e. and $\mathrm{AC}$ simultaneously, are discussed.

\section{MATERIALS AND METHODS}

\subsection{Chemicals}

Racemic mixtures of 1-phenyl-1-propanol (>97\%) (1), 1-(1naphthyl)ethanol (>99\%) (2), 1-phenylethanol (98\%) (3), $\alpha$-cyclopropylbenzyl alcohol (99\%) (4), 1-(4-methylphenyl)-1-propanol (97\%) (5), 1-phenyl-2propanol (98\%) (6), 2,2-dimethyl-1-phenyl-1-propanol (99\%) (7), $\alpha$-methyl-2naphthalenemethanol $(98 \%) \quad(8), \quad$ alpha-methyl-2-(trifluoromethyl)benzyl alcohol (9), alpha-ethyl-4-fluorobenzyl alcohol (10), were acquired from Sigma (Sigma-Aldrich Química Ltda., Chile). HPLC-grade solvents n-hexane and 2propanol were acquired from Merck (Merck S.A., Chile). All chemicals and solvents were used directly without further purification.

\subsection{Chromatographic Conditions}

Chromatographic analyses were performed on a Shimadzu HPLC chromatograph equipped with LC-20AT and LC-10ADvp pumps, a SIL-10Avp autosampler, and a CTO-10ACvp column oven. In a tandem array, a Shimadzu SPD-20A UV-vis detector $(254, \mathrm{~nm}, 10-\mathrm{mm}$ path length and $12-\mu \mathrm{L}$ internal volume flow cell), a PDR-Chemical LLR advanced laser polarimeter detector (670-nm diode laser, $25-\mathrm{mm}$ path length and $18-\mu \mathrm{L}$ internal volume flow cell), and a Jasco CD-2095 circular dichroism detector (25-mm path length and $44-\mu \mathrm{L}$ internal volume flow cell) were used in-line for UV, OR and OR measurements, respectively. The UV signal was acquired using the LCsolution software suite version 1.25 while the $\mathrm{CD}$ and OR signals were acquired with JASCO ChromNAV software version 2.01.05

Chiral separations were performed at $30{ }^{\circ} \mathrm{C}$ and a $1 \mathrm{~mL} / \mathrm{min}$ flow on Lux ${ }^{\circledR}$ Amylose-1 (150 mm, $4.6 \mathrm{~mm}, 5 \mu \mathrm{m})$, Lux ${ }^{\circledR}$ Amylose-2 $(150 \mathrm{~mm}, 4.6 \mathrm{~mm}, 3$ $\mu \mathrm{m})$, Lux ${ }^{\circledR}$ Cellulose-1 (150 mm, $\left.4.6 \mathrm{~mm}, 3 \mu \mathrm{m}\right)$, Lux ${ }^{\circledR}$ Cellulose-2 $(150 \mathrm{~mm}$, $4.6 \mathrm{~mm}, 3 \mu \mathrm{m})$, Lux ${ }^{\circledR}$ Cellulose-3 (150 mm, $\left.4.6 \mathrm{~mm}, 3 \mu \mathrm{m}\right)$ and Lux ${ }^{\circledR}$ Cellulose$4(150 \mathrm{~mm}, 4.6 \mathrm{~mm}, 3 \mu \mathrm{m})$ columns with a mobile phase composed by a mixture of n-hexane with 10, 5, 3 and $1 \%$ of 2-propanol.

Solutions of 1-10 with appropriate concentrations (between 1 and $10 \mu \mathrm{g} / \mu \mathrm{L}$ ) were prepared by dissolving in the corresponding mobile phase in $5-\mathrm{mL}$ volumetric flasks, and samples of these solutions were filtered and analysed using 1- to $20-\mu \mathrm{L}$ injections. Chiral screening was performed using $5 \mu \mathrm{g}$ injections and UV detection at $254 \mathrm{~nm}$, and leaving $30 \mathrm{~min}$ at $1 \mathrm{~mL} / \mathrm{min}$ flow between changes on mobile phase composition or CSP. Likewise, calibration curves for each compound were obtained in the 1-10 $\mu \mathrm{g}$ range using the Lux ${ }^{\circledR}$ Cellulose- 3 column using the chromatographic conditions shown in Table 2. 
Detection (DL) and quantification (QL) limits were estimated as 3.3 and 10 times the ratio of the standard deviation of $y$-intercepts and the slope of each calibration curve, as implemented in the LCsolution software suite. Also, signal to noise ratios $(\mathrm{S} / \mathrm{N})$ were measured using the signal intensity of $10 \mu \mathrm{g}$ injections and the corresponding baseline noise. This injection load was the lowest tested that produced clear signals for all compounds in the three detectors, despite the large differences in sensitivity.

\section{RESULTS AND DISCUSSION}

The chiral screening of compounds 1-10 was achieved after 240 chromatographic runs with a total runtime of over $30 \mathrm{~h}$. In each case, retention times and resolution values were recorded and are shown in Table 1.
From these values it can be seen that all six CSP showed some degree of enantioselectivity for most of the screened compounds. Nevertheless, only in some cases separations showed selectivity values higher than 1.5 , and therefore a baseline resolution capable of delivering e.e. values as required. From the CSP tested, the best performance was achieved by the 4-methylbenzoate derivative of cellulose (Lux ${ }^{\circledR}$ Cellulose-3) in which all ten compounds could be baseline resolved, followed by the 3,5-dimethylphenylcarbamate derivative of cellulose (Lux ${ }^{\circledR}$ Cellulose-1) with seven baseline resolutions. In the other hand, the worst performance of the six CSP was obtained with the 3-chloro-4methylphenylcarbamate derivatives of cellulose (Lux® Cellulose-2) and amylose (Lux ${ }^{\circ}$ Amylose-2) with only one and two baseline resolutions, respectively.

Table 1. Retention times (min) and resolutions of compounds 1-10. Resolutions over 1.5 are highlighted..

\begin{tabular}{|c|c|c|c|c|c|c|c|c|c|c|c|c|}
\hline \multirow[b]{3}{*}{ Comp } & \multicolumn{12}{|c|}{ Amylose-1 } \\
\hline & \multicolumn{3}{|c|}{ Hexane:IPA 90:10 } & \multicolumn{3}{|c|}{ Hexane:IPA 95:5 } & \multicolumn{3}{|c|}{ Hexane:IPA 97:3 } & \multicolumn{3}{|c|}{ Hexane:IPA 99:1 } \\
\hline & $\mathbf{R t}_{1}$ & $\mathbf{R t}_{2}$ & $\mathbf{R}$ & $\mathbf{R t}_{1}$ & $\mathbf{R t}_{2}$ & $\mathbf{R}$ & $\mathbf{R t}_{1}$ & $\mathbf{R t}_{2}$ & $\mathbf{R}$ & $\mathbf{R t}_{1}$ & $\mathbf{R t}_{2}$ & $\mathbf{R}$ \\
\hline 1 & 3.57 & 3.66 & 0.30 & 5.16 & 5.34 & 0.76 & 6.82 & 6.92 & 0.05 & 13.80 & - & - \\
\hline 2 & 4.90 & - & - & 7.93 & - & - & 12.02 & - & - & 29.25 & 31.41 & 1.94 \\
\hline 3 & 3.59 & - & - & 5.27 & - & - & 7.22 & 7.35 & 0.19 & 8.67 & 11.03 & 2.08 \\
\hline 4 & 4.15 & 4.30 & 0.64 & 6.29 & 6.57 & 1.11 & 8.86 & 9.21 & 1.04 & 19.31 & 19.75 & 0.39 \\
\hline 5 & 3.58 & 3.92 & 2.00 & 5.13 & 5.77 & 2.91 & 6.83 & 7.65 & 3.00 & 13.51 & 14.88 & 2.71 \\
\hline 6 & 3.32 & 3.47 & 0.92 & 4.66 & 4.95 & 1.48 & 6.26 & 6.61 & 1.44 & 12.92 & - & - \\
\hline 7 & 3.15 & - & - & 4.16 & - & - & 5.06 & - & - & 7.56 & 7.85 & 0.95 \\
\hline 8 & 5.46 & 5.64 & 0.69 & 9.09 & 9.42 & 0.92 & 14.09 & 14.67 & 0.89 & 35.55 & - & - \\
\hline 9 & 3.06 & 3.25 & 1.16 & 4.28 & 4.62 & 1.79 & 5.60 & 5.92 & 1.37 & 14.85 & - & - \\
\hline 10 & 3.51 & - & - & 5.05 & 5.16 & 0.28 & 6.97 & - & - & 14.19 & - & - \\
\hline
\end{tabular}

\begin{tabular}{|c|c|c|c|c|c|c|c|c|c|c|c|c|}
\hline & \multicolumn{12}{|c|}{ Amylose-2 } \\
\hline 1 & 3.05 & - & - & 3.93 & 4.02 & 0.20 & 5.06 & 5.19 & 0.50 & 9.51 & - & - \\
\hline 2 & 4.18 & 4.48 & 1.46 & 6.18 & 6.83 & 2.31 & 8.64 & 9.76 & 3.00 & 20.27 & 23.73 & 2.86 \\
\hline 3 & 3.13 & - & - & 4.53 & - & - & 6.02 & - & - & 12.53 & - & - \\
\hline 4 & 3.54 & 3.74 & 1.15 & 4.89 & 5.25 & 1.67 & 6.56 & 7.12 & 2.09 & 14.21 & 15.73 & 2.35 \\
\hline 5 & 3.48 & - & - & 4.00 & - & - & 5.13 & - & - & 9.31 & - & - \\
\hline 6 & 3.14 & - & - & 4.12 & - & - & 5.26 & - & - & 9.62 & 9.82 & 0.31 \\
\hline 7 & 2.59 & - & - & 3.04 & - & - & 3.53 & - & - & 5.20 & - & - \\
\hline 8 & 4.15 & - & - & 6.23 & - & - & 8.95 & - & - & 21.94 & 22.99 & 0.87 \\
\hline 9 & 2.56 & 2.64 & 0.29 & 3.13 & 3.28 & 0.90 & 3.84 & 4.07 & 1.22 & 6.75 & 7.02 & 0.83 \\
\hline 10 & 2.84 & - & - & 3.65 & - & - & 4.67 & - & - & 9.07 & - & - \\
\hline
\end{tabular}

\begin{tabular}{|c|c|c|c|c|c|c|c|c|c|c|c|c|}
\hline & \multicolumn{12}{|c|}{ Cellulose-1 } \\
\hline 1 & 3.81 & 3.99 & 1.06 & 5.64 & 5.96 & 1.43 & 7.79 & 8.48 & 2.34 & 18.25 & 21.42 & 4.66 \\
\hline 2 & 6.29 & \begin{tabular}{ll|}
10.08 \\
\end{tabular} & 11.87 & 10.74 & 18.58 & 14.75 & 16.83 & 30.35 & 16.22 & 56.16 & 100.7 & 17.04 \\
\hline 3 & 4.01 & $\begin{array}{l}4.40 \\
\end{array}$ & 2.14 & 5.97 & 6.87 & 3.78 & 8.54 & 10.26 & 5.23 & 20.58 & 27.55 & 8.70 \\
\hline 4 & 4.34 & $\begin{array}{l}4.53 \\
\end{array}$ & 1.00 & 6.63 & 6.93 & 1.17 & \begin{tabular}{|c|}
9.40 \\
\end{tabular} & 10.13 & 2.07 & 22.67 & 27.31 & 5.29 \\
\hline 5 & 3.60 & - & - & 5.10 & - & - & 6.96 & - & - & 15.37 & 16.23 & 1.63 \\
\hline 6 & 3.46 & 3.67 & 1.34 & 4.75 & 5.17 & 2.08 & 6.29 & 6.99 & 2.81 & 13.25 & 15.31 & 4.18 \\
\hline 7 & 3.30 & 4.30 & \begin{tabular}{|l|}
5.78 \\
\end{tabular} & 4.41 & 6.27 & 8.52 & \begin{tabular}{|l|l|}
5.65 \\
\end{tabular} & 8.31 & 9.78 & 11.34 & 16.69 & 9.77 \\
\hline 8 & 6.92 & 7.08 & 0.36 & 12.50 & - & - & 19.70 & 20.00 & 0.13 & 64.99 & 66.28 & 0.32 \\
\hline 9 & 2.90 & - & - & 3.89 & - & - & 5.12 & - & - & 11.47 & - & - \\
\hline 10 & 3.32 & - & - & 4.59 & 4.72 & 0.55 & 6.21 & 6.42 & 0.85 & 14.29 & 14.96 & 1.30 \\
\hline
\end{tabular}

\begin{tabular}{|c|c|c|c|c|c|c|c|c|c|c|c|c|}
\hline & \multicolumn{12}{|c|}{ Cellulose-2 } \\
\hline 1 & 3.14 & - & - & 4.47 & - & - & 6.00 & - & - & 15.09 & - & - \\
\hline 2 & 4.03 & 4.55 & 0.83 & 6.36 & 7.47 & 1.05 & 9.21 & 11.03 & 1.13 & 27.67 & 33.97 & 0.14 \\
\hline 3 & 3.48 & - & - & 4.85 & 5.28 & 0.51 & 6.72 & 7.42 & 0.56 & 18.17 & 20.37 & 0.99 \\
\hline 4 & 3.84 & - & - & 5.82 & - & - & 8.32 & - & - & 23.00 & - & - \\
\hline 5 & 3.00 & - & - & 4.43 & - & - & 6.00 & - & - & 15.18 & - & - \\
\hline 6 & 3.10 & - & - & 4.23 & - & - & 5.58 & - & - & 12.71 & - & - \\
\hline 7 & 2.50 & 2.85 & 1.06 & 3.05 & 3.69 & 1.54 & 3.67 & 4.61 & 1.76 & 7.96 & 10.00 & 1.53 \\
\hline 8 & 4.20 & 4.42 & 0.06 & 6.71 & 7.21 & $\begin{array}{l}0.29 \\
\end{array}$ & 10.20 & 11.04 & 0.31 & 39.60 & 41.58 & 0.16 \\
\hline 9 & 2.47 & - & - & 3.17 & - & - & 3.86 & 4.06 & 0.08 & 10.21 & - & - \\
\hline 10 & 2.90 & - & - & 4.01 & - & - & 5.45 & - & - & 16.51 & - & - \\
\hline
\end{tabular}

\begin{tabular}{|c|c|c|c|c|c|c|c|c|c|c|c|c|}
\hline & \multicolumn{12}{|c|}{ Cellulose-3 } \\
\hline 1 & 3.71 & 3.87 & 0.97 & 5.11 & 5.44 & 1.64 & 6.85 & 7.35 & 1.91 & 12.82 & 13.16 & 0.57 \\
\hline 2 & 6.69 & 9.03 & 8.13 & 11.13 & 16.53 & 11.04 & 16.53 & 26.42 & 13.19 & 28.89 & 56.32 & 17.45 \\
\hline 3 & 4.02 & 4.37 & 2.12 & 5.81 & 6.52 & 3.18 & 8.12 & 9.21 & 3.52 & 16.79 & 17.61 & 1.34 \\
\hline 4 & 4.74 & 5.17 & 2.20 & $\begin{array}{l}7.08 \\
\end{array}$ & 7.95 & 3.12 & 9.99 & 11.33 & 3.07 & 18.73 & 21.01 & 2.72 \\
\hline 5 & 3.64 & 3.73 & 0.43 & 5.02 & 5.23 & 1.06 & 6.80 & 7.01 & 0.76 & 12.31 & 13.05 & 1.70 \\
\hline 6 & 3.49 & - & - & 4.76 & - & - & 6.27 & 6.41 & 0.35 & 10.82 & 11.45 & 1.50 \\
\hline 7 & 3.30 & - & - & 4.18 & - & - & 4.97 & - & - & 5.81 & 6.37 & 1.73 \\
\hline 8 & 7.86 & 9.79 & 6.19 & 13.91 & 18.06 & $\begin{array}{l}7.68 \\
\end{array}$ & 21.73 & 28.58 & 8.18 & 53.00 & 68.48 & 7.65 \\
\hline 9 & 2.73 & 2.91 & 1.20 & 3.41 & 3.71 & 1.85 & 4.19 & 4.62 & 2.23 & 7.88 & 8.27 & 1.07 \\
\hline 10 & 3.56 & - & - & 4.97 & - & - & 6.65 & - & - & 12.07 & 13.24 & 2.44 \\
\hline
\end{tabular}

\begin{tabular}{|c|c|c|c|c|c|c|c|c|c|c|c|c|}
\hline & \multicolumn{12}{|c|}{ Cellulose-4 } \\
\hline 1 & 2.97 & - & - & 3.89 & 4.02 & 0.52 & 5.06 & 5.29 & 0.97 & 11.52 & 12.55 & 2.02 \\
\hline 2 & 3.94 & 4.14 & 1.04 & 6.03 & 6.46 & 1.60 & 8.69 & 9.45 & 2.01 & 24.65 & 27.89 & 3.15 \\
\hline 3 & 3.17 & 3.28 & 0.68 & 4.36 & 4.64 & 1.37 & 5.91 & 6.38 & 1.81 & 14.61 & 16.30 & 2.76 \\
\hline 4 & 3.44 & - & - & 4.85 & 5.00 & 0.51 & 6.60 & 6.88 & 0.93 & 16.58 & 18.04 & 1.90 \\
\hline 5 & 3.01 & - & - & 4.03 & - & - & 5.29 & - & - & 12.28 & - & - \\
\hline 6 & 2.98 & - & - & 3.87 & - & - & 4.95 & - & - & 10.40 & - & - \\
\hline 7 & 2.50 & - & - & 2.95 & - & - & 3.45 & - & - & 5.86 & 6.21 & 1.29 \\
\hline 8 & 4.11 & - & - & 6.37 & 6.52 & 0.27 & 9.49 & 9.73 & 0.41 & 32.25 & 33.19 & 0.53 \\
\hline 9 & 2.46 & - & - & 2.98 & - & - & 3.68 & - & - & 7.58 & 7.92 & 0.80 \\
\hline 10 & 2.81 & - & - & 3.67 & - & - & 4.78 & - & - & 11.18 & - & - \\
\hline
\end{tabular}


Furthermore, the effect of reducing the concentration of the polar mobile phase modifier (2-propanol) was in most cases the one expected in normal phase chiral chromatography; an increase in retention times along with a better resolution of the enantiomers in the mixture. Nevertheless, in many cases this trend was reversed at low IPA concentrations, in which a loss of resolution and sometimes peak distortion was observed. This is the case of compounds $\mathbf{1 , 3}$ and 9 when tested on Celullose-3, which can be efficiently resolved at 3\% IPA but not at $1 \%$. The above is probably due to the low solubility of these compounds in the more highly aliphatic mobile phases.
Table 2 shows the estimated runtimes for the separations of compounds 110 using the Lux ${ }^{\circledR}$ Cellulose-3 CSP and the fastest mobile phase for each compound. Each runtime was calculated adding $30 \mathrm{~s}$ after the complete elution of the second enantiomer. From these values, and considering a $30 \mathrm{~min}$ equilibration period between mobile phase changes, a total runtime of $148 \mathrm{~min}$ could be estimated for the entire analysis. Additionally, detection (DL) and quantification $(\mathrm{QL})$ limits were estimated for each chiral resolution using UV detection at $254 \mathrm{~nm}$.

Table 1. Estimated runtimes ( $\mathrm{min}$ ), mobile phase composition, elution order, detection and quantification limits and signal to noise ratios of resolutions for compounds 1-10 in 4-methylbenzoate derivative of cellulose (Lux® Cellulose-3) CSP.

\begin{tabular}{|c|c|c|c|c|c|c|c|c|c|c|}
\hline Comp & RunT (min) & IPA & $\mathrm{OR}^{\mathrm{a}}$ & $\mathrm{CD}^{\mathrm{a}}$ & $\mathrm{AC}^{\mathbf{b}}$ & $\mathrm{DL}(\mu \mathrm{g})^{\mathrm{c}}$ & $\mathrm{QL}(\mu \mathrm{g})^{\mathrm{c}}$ & $\mathbf{S} / \mathbf{N}(\mathbf{U V})^{\mathbf{c}}$ & $\mathrm{S} / \mathrm{N}(\mathrm{OR})^{\mathrm{d}}$ & $\mathrm{S} / \mathrm{N}(C D)^{\mathrm{e}}$ \\
\hline 1 & 6.2 & $5 \%$ & $-/+$ & $+/-$ & $S / R$ & $1.5 \times 10^{-1}$ & $4.6 \times 10^{-1}$ & $2.0 \times 10^{+4}$ & $1.8 \times 10^{+1}$ & $6.2 \times 10^{+1}$ \\
\hline 2 & 9.9 & $10 \%$ & $-/+$ & $-1+$ & $S / R$ & $4.8 \times 10^{-2}$ & $1.5 \times 10^{-1}$ & $2.6 \times 10^{+4}$ & $1.0 \times 10^{+1}$ & $7.0 \times 10^{+1}$ \\
\hline 3 & 5.1 & $10 \%$ & $-/+$ & $+/-$ & $S / R$ & $4.6 \times 10^{-2}$ & $1.4 \times 10^{-1}$ & $3.8 \times 10^{+3}$ & $1.8 \times 10^{+1}$ & $2.2 \times 10^{+1}$ \\
\hline 4 & 6.0 & $10 \%$ & $+/-$ & $+/-$ & $S / R$ & $4.7 \times 10^{-2}$ & $1.4 \times 10^{-1}$ & $4.0 \times 10^{+3}$ & 3.8 & $1.7 \times 10^{+1}$ \\
\hline 5 & 5.2 & $1 \%$ & $+/-$ & $-/+$ & $R / S$ & $6.7 \times 10^{-2}$ & $2.0 \times 10^{-1}$ & $1.3 \times 10^{+3}$ & $1.3 \times 10^{+1}$ & $1.1 \times 10^{+1}$ \\
\hline 6 & 12.8 & $1 \%$ & $-/+$ & $-1+$ & $R / S$ & $5.2 \times 10^{-2}$ & $1.6 \times 10^{-1}$ & $1.5 \times 10^{+3}$ & 8.6 & $1.3 \times 10^{+1}$ \\
\hline 7 & 7.4 & $1 \%$ & $-/+$ & $+/-$ & $S / R$ & $6.2 \times 10^{-2}$ & $1.9 \times 10^{-1}$ & $2.0 \times 10^{+3}$ & $1.3 \times 10^{+1}$ & $4.2 \times 10^{+1}$ \\
\hline 8 & 11.0 & $10 \%$ & $-/+$ & $+/-$ & $S / R$ & $4.4 \times 10^{-2}$ & $1.3 \times 10^{-1}$ & $7.6 \times 10^{+4}$ & $2.8 \times 10^{+2}$ & $4.8 \times 10^{+2}$ \\
\hline 9 & 4.5 & $5 \%$ & $-/+$ & $+/-$ & $S / R$ & $3.0 \times 10^{-1}$ & $9.0 \times 10^{-1}$ & $7.6 \times 10^{+3}$ & $2.2 \times 10^{+1}$ & $4.4 \times 10^{+1}$ \\
\hline 10 & 14.8 & $1 \%$ & $+/-$ & $-/+$ & $R / S$ & $1.7 \times 10^{-1}$ & $5.2 \times 10^{-1}$ & $3.0 \times 10^{+3}$ & 9.4 & 2.2 \\
\hline
\end{tabular}

${ }^{a}$ sign of the chiroptical effect in order of elution. ${ }^{\mathrm{b}}$ absolute configurations in order of elution and deduced from literature data; $\mathbf{2}, \mathbf{3}, \mathbf{9}$ ref. $6, \mathbf{1}$ ref. $11, \mathbf{8}$ ref. $12, \mathbf{4}$ ref. $13, \mathbf{5}, \mathbf{1 0}$ ref. 14,7 ref. $15, \mathbf{6}$ ref. $16 .{ }^{\mathrm{c}}$ measured at $254 \mathrm{~nm} .{ }^{\mathrm{d}}$ measured at $670 \mathrm{~nm} .{ }^{\mathrm{e}}$ measured at $230 \mathrm{~nm}$ for $\mathbf{6}, 7, \mathbf{1 0} ; 240 \mathrm{~nm}$ for $\mathbf{2}, \mathbf{3}, \mathbf{4}, \mathbf{5} ; 250$ $\mathrm{nm}$ for $\mathbf{1}$ and $\mathbf{9}$; and $300 \mathrm{~nm}$ for $\mathbf{8}$.

Furthermore, the addition of the laser polarimetric detector in series to the UV detector showed the optical activity of the eluted peaks, revealing the elution order in each chromatographic run (Table 2), as showed for compound 2 (Figure 2). Each of these optical rotation signs can easily be related to a particular AC through literature data ${ }^{6,11-16}$, which allows the full characterization of the sample through the HPLC analysis. This avoids the need for conventional polarimetric measurements and considerably decreases the time to screen the stereoselectivity of new catalyst during the development phase. While it is possible to infer the $\mathrm{AC}$ of the preferred enantiomer solely from the elution order of the peaks, this is generally not recommended since reversal of elution can occur, particularly when the column temperature is not well controlled ${ }^{17}$. To avoid this issue, the simpler option is to use enantiomerically pure standards of the compounds to unequivocally assign the $\mathrm{AC}$ of the isomer in excess, although this will double the number of injections needed to complete the batch analysis.

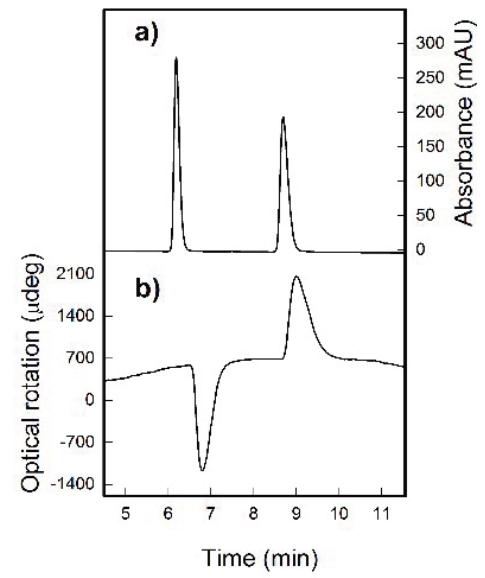

Fig. 2. Chiral separation of compound 2. (a) UV detection at $254 \mathrm{~nm}, 7.1 \mu \mathrm{g}$ injection. (b) OR detection at $670 \mathrm{~nm}, 105.2 \mu \mathrm{g}$ injection.

On the other hand, the use of the laser polarimeter as a second detector, along with the UV detector, will provide the AC of the major enantiomer directly without standards. Nonetheless, it is important to notice that a considerable difference in signal to noise ratios $(\mathrm{S} / \mathrm{N})$ between $\mathrm{UV}$ and polarimetric detections will become evident during the analyses. This is due the particularly low sensitivity of the optical rotation measurement when compared to UV-vis absorption, which in most cases produces a difference in $\mathrm{S} / \mathrm{N}$ values between both detections of two or even 3 orders of magnitude (Table 2). In consequence, a larger amount of sample (often between 50 to $100 \mu \mathrm{g}$ ) needs to be injected on the chromatograph in order to obtain a clear signal from the polarimeter. While these sample amounts represent only a fraction of what is needed for conventional polarimetric measurements, such large injection loads can saturate the UV detector if an analytical flow cell is used. This can be overcome by selecting a different wavelength for the UV detector or using a semipreparative flow cell with a larger light path to avoid the saturation on the photomultiplier at these high sample loads. Nonetheless, one should be aware of potential loss of resolution or even peak overlapping at these chromatographic conditions. Alternatively, two separate injections of the same solution with different volumes (e.g. 1 and $50 \mu \mathrm{L}$ of a $1 \mathrm{mg} / \mathrm{mL}$ solution), or different concentration solutions with the same volume (e.g. $25 \mu \mathrm{L}$ of a 2 and 0.1 $\mathrm{mg} / \mathrm{mL}$ solutions), will suffice to obtain both UV and OR chromatograms for each compound. However, as with the use of enantiomerically pure standards, this will double the injections in the batch and the total analysis time.

Similarly, a complete sample analysis that includes both e.e. and AC assessment can also be achieved using another chiral HPLC detector, this time based on the electronic CD effect. Using this option, both UV and CD signals can be acquired simultaneously within the same detector, and in some cases without separate injections for the same compound due to the closer magnitude between both signals. On the other hand, while the sign of CD signals at a given wavelength can be related to a particular enantiomer in the racemic mixture, $C D$ information is much harder to find in the literature compared to optical rotation values. This is critical since without a reference CD spectrum, it would be impossible to establish the stereochemical preference of the reaction sample from the CD peak alone. Nevertheless, in this case reference CD spectra are not necessary since the previous use the OR detection already established the AC of each chromatographic peak. Considering this, Table 2 shows the signs of optical rotation (measured at $670 \mathrm{~nm}$ ) and CD signals (measured at $230 \mathrm{~nm}$ for $\mathbf{6}$, $\mathbf{7}, \mathbf{1 0} ; 240 \mathrm{~nm}$ for $\mathbf{2}, \mathbf{3}, \mathbf{4}, \mathbf{5} ; 250$ for $\mathbf{1}$ and $\mathbf{9}$; and $300 \mathrm{~nm}$ for $\mathbf{8}$ ) for the first and second eluting enantiomer of each compound, along with the corresponding AC. 
Considering all of the above, at this time is possible to propose several different methodologies that can be applied to analyse samples from the asymmetric reduction of ketones that leads to the set of aromatic alcohols described herein. In the simpler approach, the samples can be analysed using the single-column bath procedure already described using only the HPLC-UV chromatograph. From the UV chromatogram, it will be possible to obtain the e.e. from the peaks area, and the stereochemical preference of the reaction must be assigned using the elution order of the peaks. Alternatively, a more reliable assignment can be done without additional chromatographic hardware using enantiomerically pure standards of each compounds if available, but not without adding a considerable amount of time to the total batch time. As a second approach, the batch procedure can be run using an HPLC-UV-OR arrangement, from which e.e. and $\mathrm{AC}$ can be obtained from the UV an OR chromatograms directly without standards. Nevertheless, as stated above, the difference in sensitivities between both detectors will require the use of a less sensitive wavelength for the UV detector, a semipreparative flow cell, or the separate injection of different amounts of sample, to obtain the needed data for both signals. Finally, a third approach can be proposed using an HPLC-CD chromatograph in which both signals (UV and CD) are received simultaneously from the same detector. In both chiroptical detections, the information gathered herein should suffice to assign the stereochemical preference of the reaction without the need for enantiomerically pure standards.

\section{CONCLUSIONS}

The evaluation of a newly developed catalyst for the asymmetric reduction of ketones to chiral aromatic alcohols is a time-consuming process that can be greatly improved by a more efficient analytical methodology. With this in mind, the chiral HPLC-UV screening of ten racemic aromatic alcohols on six modified polysaccharide-based CSP was performed under normal phase mode. While all CSP showed some degree of enantioselectivity for most of the screened compounds, one of them (Lux $®$ Cellulose-3) stood out for being able to resolve all tested racemates, although at different chromatographic conditions. This finding allowed us to propose a single-column batch analysis that is capable of delivering the enantiomer excesses of all tested samples. Furthermore, since the reaction outcome also needs a stereochemical characterization of the preferred enantiomer, a second detector based on optical rotation measurements was used to assign the AC of both chromatographic peaks. From these assignments, or using enantiomerically pure standards, it is possible to obtain both necessary parameters (e.e. and AC) to evaluate the catalyst performance for each compound. As a second option, the optical rotation detector can be used directly to reliably assign the $\mathrm{AC}$ of the preferred isomer, although a fine-tuning of the UV wavelength, a semipreparative UV flow cell or dual injections of different concentration will be needed to account for the large difference in sensitivity between both detectors. Finally, a third option is proposed by using a CD detector to acquire UV and CD chromatograms, from which e.e. and AC can also be obtained without any enantiomerically pure standards.

\section{ACKNOWLEDGEMENT}

The authors acknowledge financial support from CONICYT-Chile (project FONDECYT 1141071)

\section{REFERENCES}

1. V. Farina, J.T. Reeves, C.H. Senanayake, J.J. Song, Chem. Rev. 106, 2734 2793, (2006)

2. N. Vergesson, Birth Defect Res C. 105, 140-156, (2015)

3. A. Calcaterra, I. D'Acquarica, J. Pharm. Biomed. Anal. 147, 323-340, (2018)

4. B. Štefane, F. Požgan, Catalysis Reviews 56, 82-174, (2014)

5. B. Štefane, F. Požgan, Topics in Current Chemistry 374, 18, (2016)

6. H. Shimizu, D. Igarashi, W. Kuriyama, Y. Yusa, N. Sayo, T. Saito, Org. Lett. 9, 1655-1657, (2007)

7. D. Zhu, Y. Yang, L. Hua, J. Org. Chem. 71, 4202-4205, (2006)

8. L. Kott, W.B. Holzheuer, M.M. Wong, G.K. Webster, J. Pharm. Biomed. Anal. 43, 57-65, (2007)

9. I. Ali, H.Y. Aboul-Enein, Role of polysachccharides in chiral separations by liquid chromatography and capillary electrophoresis, in: G. Subramanian (Ed.), Chiral Separation Techniques. A Practical Approach, 3nd ed., WileyVCH: Weinheim, 2007; pp. 29-97.

10. H.Srour, P. Le Maux, G. Simonneaux, Inorg. Chem. 51, 5850-5856, (2012)

11. D.R. Li, A. He, J.R. Falck, Org. Lett. 12, 1756-1759, (2010)
12. B.K. Langlotz, H. Wadepohl, L.H. Gade, Angew. Chem. Int. Ed. 47, 46704674, (2008)

13. T. Ohkuma, M. Koizumi, H. Doucet, T. Pham, M. Kozawa, K. Murata, E. Katayama, T. Yokozawa, T. Ikariya, R. Noyori, J. Am. Chem. Soc. 120, 13529-13530, (1998)

14. Š. Vyskočil, S. Jaracz, M. Smrčina, M. Štícha, V. Hanuš, M. Polášek, P. Kočovský, J. Org. Chem. 63, 7727-7737, (1998)

15. J. Chandrasekharan, P.V. Ramachandran, H.C. Brown, J. Org. Chem. 50, 5446-5448, (1985)

16. M.M. Musa, K.I. Ziegelmann-Fjeld, C. Vieille, J.G. Zeikus, R.S. Phillips, J. Org. Chem. 72, 30-34, (2007)

17. X. Yang, L. Su, X. Hou, S. Ding, W. Xu, B. Wang, H. Fang, J. Chromatogr. $A, \mathbf{1 3 5 5}, 291-295,(2014)$ 